\title{
O padrão entoacional das sentenças interrogativas do português brasileiro em fala manipulada
}

\author{
Carolina Carbonari Rosignoli \\ Universidade de São Paulo (USP), São Paulo, São Paulo, Brasil \\ carolina.rosignoli@usp.br \\ Flaviane Romani Fernandes-Svartman \\ Universidade de São Paulo (USP), São Paulo, São Paulo, Brasil \\ flavianesvartman@usp.br
}

DOI: $\underline{\text { http://dx.doi.org/10.21165/el.v45i1.1449 }}$

\begin{abstract}
Resumo
$\mathrm{O}$ presente artigo pretende investigar padrões entoacionais em enunciados interrogativos globais do dialeto paulista do português brasileiro, de modo a observar diferenças e semelhanças prosódicas que definam os diferentes tipos frásicos de enunciados interrogativos globais. Este trabalho é fundamentado pela teoria da Fonologia Entoacional (PIERREHUMBERT, 1980; PIERREHUMBERT; BECKMAN, 1988; LADD, 1996, 2008; JUN, 2005), segundo a qual, levando-se em conta um conjunto de características suprassegmentais das sentenças, é possível interpretar significados pragmáticos de uma maneira linguisticamente organizada. A partir de um corpus inédito e em acréscimo às pesquisas de Frota et al. (2015) e Moraes (2008), os resultados da presente pesquisa revelam que variações dos contornos nuclear e total são distintivas na configuração prosódica de cada tipo frásico.
\end{abstract}

Palavras-chave: português brasileiro; prosódia; sentenças interrogativas globais.

\section{The Intonational Pattern of Interrogative Sentences in Brazilian Portuguese in Manipulated Speech}

\begin{abstract}
This paper aims to investigate intonational patterns in global interrogative sentences belonging to the Paulista dialect, spoken in Brazil, in order to observe prosodic distinctions and similarities that can define different clause types of global interrogative sentences. This study is based on Intonational Phonology (PIERREHUMBERT, 1980; PIERREHUMBERT; BECKMAN, 1988; LADD, 1996, 2008; JUN, 2005), which proposes that it is possible to interpret pragmatical meanings in a linguistically organized manner, given a set of suprasegmental characteristics of sentences. Based on an original corpus and in addition to Frota et al. (2015) and Moraes (2008), the results of this research reveal that variations in the nuclear and total contours are distinctive in the prosodic configuration of each clause type.
\end{abstract}

Keywords: Brazilian Portuguese; prosody; global interrogative sentences. 


\section{Introdução}

O presente artigo visa à investigação dos padrões entoacionais associados a sentenças interrogativas globais do português, as quais, conforme classificação sugerida por Mateus et al. (2003), correspondem às perguntas em que se espera como resposta "sim" ou "não", conforme o exemplo em (01):

O João foi à festa?

Neste artigo, temos como objetivo específico a descrição suprassegmental da curva melódica encontrada em frases interrogativas globais da variedade paulista do português brasileiro (doravante, PB), a partir do sistema descritivo sugerido pela Fonologia Entoacional (ver seção "Quadro teórico e trabalhos prévios" deste artigo).

Pretendemos identificar possíveis contrastes na curva entoacional em função de diferenças entre os tipos frásicos interrogativos (ver subseção "Classificação dos tipos frásicos" deste artigo), diferenças essas que dependem de contextos extralinguísticos, revelando conteúdos específicos que podem aparecer refletidos na entoação, conforme será discutido a seguir.

Este artigo se encontra organizado da seguinte maneira: (i) na seção "Quadro teórico e trabalhos prévios", apresentaremos o quadro teórico no qual se ancora este trabalho e os resultados obtidos previamente por outros autores sobre o mesmo tema deste artigo; (ii) na seção "Materiais e métodos", apresentaremos os corpora empregados na investigação aqui apresentada e a metodologia aplicada na coleta, análise e interpretação dos dados; (iii) já na seção "Análise de dados e discussão", serão apresentados os resultados obtidos da análise de dados, bem como a discussão desses resultados; e (iv) por sua vez, na seção "Considerações finais", apresentaremos nossas conclusões.

\section{Quadro teórico e trabalhos prévios}

Este trabalho é fundamentado pela teoria da Fonologia Entoacional (PIERREHUMBERT, 1980; PIERREHUMBERT; BECKMAN, 1988; LADD, 1996, 2008; JUN, 2005), segundo a qual, levando-se em conta um conjunto de características suprassegmentais das sentenças é possível interpretar significados pragmáticos de uma maneira linguisticamente organizada. Em tal teoria, é proposta uma descrição universal para a entoação e seu pressuposto básico é que há uma organização fonológica da entoação. Dessa forma, a entoação é tratada a partir de contornos entoacionais que são uma sequência de unidades chamadas de eventos tonais, alocados em pontos específicos da cadeia segmental (PIERREHUMBERT, 1980; PIERREHUMBERT; BECKMAN, 1988, entre outros). Para Cruz e Frota (2011), os eventos tonais de uma língua comportam-se como morfemas que codificam informações semânticas e pragmáticas, seja isoladamente ou em sequência. A representação fonética da sequência de eventos tonais consiste no contorno da frequência fundamental $\left(\mathrm{F}_{0}\right)$. 
De acordo com a Fonologia Entoacional, os eventos tonais definem-se em contraste uns com os outros quanto ao critério da altura. Atribuem-se os símbolos $\mathrm{H}$ (high) para tons altos e L (low) para tons baixos, entre outras denotações para eventos tonais específicos. Há, por exemplo, a marcação de tom mais alto, numa mesma sentença, em relação a um outro tom alto imediatamente anterior (com a representação de upstep " $i$ " antecedendo " $\mathrm{H}$ " "degrau acima") e a marcação de tom mais baixo em relação a um tom alto imediatamente anterior, mas ainda assim alto, dentro da sentença (com a representação de downstep "!" antecedendo "H”- "degrau abaixo").

Os eventos tonais ainda podem ser simples (monotonais: $\mathrm{H}$ ou $\mathrm{L}$ ) ou complexos (bitonais: LH ou HL) e compreendem dois tipos: os acentos tonais e os tons relacionados a fronteiras. Os acentos tonais são associados a sílabas proeminentes na cadeia segmental e são representados pelo alvo de altura (H, L, LH ou HL) seguido de asterisco (*). Os tons relacionados a fronteiras são os tons de fronteira (boundary tones: $\mathrm{L} \%, \mathrm{H} \%, \mathrm{HL} \%$ ou LH\%), relacionados a fronteiras de constituintes maiores (por exemplo, fronteira de fim de enunciado), e os acentos frasais (phrasal accents: $\mathrm{L}^{-}$ou $\mathrm{H}^{-}$- conforme a notação de Pierrehumbert), ${ }^{1}$ relacionados a fronteiras de constituintes mais baixos (por exemplo, fronteira de fim de sintagma).

Quanto aos trabalhos prévios sobre o padrão melódico de interrogativas em PB desenvolvidos à luz da Fonologia Entoacional, destacamos os trabalhos de Moraes (2008), Truckenbrodt, Sandalo e Abaurre (2009) e Frota et al. (2011, 2015).

As pesquisas de Moraes (2008) e Frota et al. $(2011,2015)$ apontam que o contorno entoacional padrão para interrogativas globais, de maneira geral, pode ser definido como $\mathrm{L}^{*}+\mathrm{H}$ L\% para PB. Frota et al. $(2011,2015)$ ainda apontam que, em PB, o tom de fronteira pode variar nas interrogativas, sendo ora $\mathrm{H} \%$ ora $\mathrm{L} \%$, especificando que, para a variedade paulista, o tom de fronteira identificado como tendência é baixo (L\%), enquanto que, por exemplo, para as variedades baiana e potiguar, o tom de fronteira é frequentemente alto $(\mathrm{H} \%)$.

Truckenbrodt, Sandalo e Abaurre (2009) confirmam o padrão ascendente apontado inicialmente por Moraes, afirmando que o padrão entoacional nuclear de interrogativas simnão (yes-no questions) pode ser definido como $\mathrm{L}+\mathrm{H}^{*} \mathrm{~L} \%$. Na descrição proposta por Truckenbrodt, Sandalo e Abaurre, o acento tonal é distinto do apontado anteriormente $\left(\mathrm{L}^{*}+\mathrm{H}\right)$ como padrão para as interrogativas globais, de maneira geral, em PB. Os autores também descrevem o padrão entoacional de perguntas surpresa (surprise questions), definindo-o como $\mathrm{L}^{*}+\mathrm{H} \mathrm{L} \%$. Esse tipo de interrogativas é classificado neste artigo como interrogativa não neutra antiexpectativa (ver subseção "Classificação dos tipos frásicos").

Neste artigo, trataremos não só da descrição entoacional do contorno nuclear, mas também da descrição entoacional do contorno total dos enunciados interrogativos globais. Nossa hipótese é que o padrão entoacional global dos enunciados interrogativos, e não só o contorno nuclear, é relevante na caracterização dos diferentes tipos frásicos.

\footnotetext{
${ }^{1} \mathrm{Na}$ literatura linguística brasileira sobre entoação do PB, "acento frasal" é o termo mais utilizado para a tradução de phrasal accent.
} 


\section{Materiais e métodos}

Nesta seção, serão dadas informações detalhadas sobre os corpora e sobre os procedimentos metodológicos empregados no desenvolvimento deste trabalho.

\section{Corpora}

Os dados selecionados para o desenvolvimento de nossa pesquisa são: (i) de um corpus de leitura controlada, contemplando enunciados interrogativos globais e (ii) de corpora de fala do banco de dados do projeto "Atlas Interactivo da Prosódia do Português". 2

O corpus especificado em (i) possui 36 enunciados interrogativos globais, sendo quatro por tipo frásico de interrogativa global delimitado conforme a classificação apresentada no Quadro 1 a seguir. Na elaboração desse corpus, foram escolhidas palavras paroxítonas e proparoxítonas para ocupar a posição nuclear nas frases analisadas, de maneira a resguardar informação sonora para além da silaba tônica nuclear, facilitando a identificação dos contornos nuclear e de fronteira. Para cada tipo frásico, foram formuladas quatro frases, sendo duas com palavra nuclear paroxítona e duas com palavra nuclear proparoxítona.

Quanto aos corpora especificados em (ii), referem-se a dados contendo enunciados de diversos tipos (interrogativos globais e parciais, neutros, exclamativos, imperativos, focalizados) e obtidos a partir de diferentes contextos de produção: dados de fala controlada (obtidos a partir de leitura), dados de fala semicontrolada (obtidos através de aplicação de questionários direcionados e através de execução de tarefa de indicação de direções em mapas pelos falantes). Desses corpora, foram analisados, em nosso trabalho, apenas os enunciados interrogativos globais.

\section{Classificação dos tipos frásicos}

A classificação de sentenças interrogativas, adaptada para o desenvolvimento deste trabalho, levou em conta os tipos frásicos apresentados pelo projeto InAPoP e pelo trabalho de Mateus et al. (2003). Definiu-se a nomenclatura especificada no Quadro 1 para fins de análise e classificação das sentenças interrogativas globais. Cabe acrescentar que, conforme apresentado no Quadro 1, entende-se por interrogativas globais neutras aquelas que não apresentam uma resposta esperada ao que se pergunta (ver exemplos no Quadro 1). As interrogativas globais não neutras, por sua vez, apresentam uma expectativa de resposta (ver exemplos no Quadro 1).

\footnotetext{
2 O projeto "Atlas Interativo da Prosódia do Português" (InAPoP), ao qual este trabalho se vincula, é coordenado pela Profa. Dra. Sónia Frota e se encontra em desenvolvimento na Universidade de Lisboa. Tal projeto tem como principal objetivo a construção de um atlas interativo da prosódia do português, acessado livremente por uma plataforma online (http://www.fl.ul.pt/LaboratorioFonetica/InAPoP/), que contemple a variação prosódica, entoacional e rítmica do português, incluindo a cobertura completa do português europeu (doravante, PE) quanto a esses três aspectos, contanto ainda com variedades do PB ao longo da costa do Atlântico, assim como variedades do português falado na África.
} 
Quadro 1. Classificação dos tipos frásicos de interrogativas globais.

\begin{tabular}{|c|c|l|l|}
\hline $\begin{array}{c}\text { Tipos } \\
\text { Frásicos }\end{array}$ & Sigla $^{3}$ & Especificação & Exemplo \\
\hline \multirow{5}{*}{ Neutras } & GNI & Global Neutra de Busca de Informações & O João vai à festa? \\
\cline { 2 - 4 } & GND & Global Neutra Disjuntiva & $\begin{array}{l}\text { Você vai ao mercado ou à } \\
\text { farmácia? }\end{array}$ \\
\cline { 2 - 5 } & GNR & Global Neutra Retórica & Será que eu fiz a melhor escolha? \\
\hline \multirow{5}{*}{$\begin{array}{c}\text { Não } \\
\text { neutras }\end{array}$} & GAF & Global Não-Neutra Focalizada & $\begin{array}{l}\text { É amanhã que você vai à } \\
\text { entrevista? }\end{array}$ \\
\cline { 2 - 5 } & GAN & Global Não-Neutra Negativa (manipulatória) $)^{4}$ & Você não vai estudar? \\
\cline { 2 - 5 } & GAT & Global Não-Neutra Confirmatória & É aqui mesmo o local da festa? \\
\cline { 2 - 4 } & GAE & Global Não-Neutra Eco Absoluta & Entendeu? / Concorda? \\
\cline { 2 - 4 } & GAA & $\begin{array}{l}\text { Global Não-Neutra Antiexpectativa } \\
\text { (incredulidade) }\end{array}$ & O quê? / Como disse? \\
\hline
\end{tabular}

\section{Coleta de dados}

Os dados relativos à fala controlada foram coletados por meio de gravação realizada com gravador TASCAM H2-P2 (Frontier) e microfone de lapela Sennheiser, contando com três informantes mulheres, entre 20 e 40 anos, com ensino superior completo, paulistas, que repetiram cada sentença do corpus três vezes, totalizando 324 enunciados produzidos.

Os dados relativos aos corpora do projeto InAPoP foram coletados anteriormente, obtidos por meio de gravação, realizada com gravador MARANTZ e microfone de lapela Sennheiser, da produção de falantes com as mesmas características das participantes da coleta anterior (mulheres, entre 20 e 40 anos, com ensino superior completo e paulistas).

\section{Análise de dados e discussão}

Os enunciados interrogativos foram submetidos à análise prosódica, que consistiu na transcrição e na descrição de todos os eventos tonais associados ao contorno entoacional de cada enunciado.

A transcrição dos eventos tonais associados ao contorno entoacional dos enunciados interrogativos foi realizada através do uso do software Praat (BOERSMA; WEENINK, 2014) e com base nos pressupostos teóricos da Fonologia Entoacional (ver seção "Quadro teórico e trabalhos prévios" deste artigo) e, especificamente para o português do Brasil, FROTA; VIGÁRIO, 2000; TENANI, 2002; FERNANDES, 2007a, 2007b; SERRA, 2009; TRUCKENBRODT; SANDALO; ABAURRE, 2009; VIGÁRIO; FERNANDESSVARTMAN, 2010).

\footnotetext{
${ }^{3}$ Foram elaboradas siglas para catalogação dos dados e do corpus de modo a facilitar o trabalho de análise, tratamento e discussão dos dados. Por exemplo, a sigla G representa as interrogativas globais. A sigla $\mathrm{N}$ representa as neutras, enquanto que a sigla A foi escolhida para representar as não neutras, para criar um contraste em relação à sigla das interrogativas neutras.

${ }^{4}$ A classificação das Negativas foi extraída do trabalho de Mateus et al. (2003), de modo a complementar a clas sificação utilizada pelo projeto InAPoP.
} 
A seguir, na Tabela 1, é possível observar tendências de padrões entoacionais do contorno nuclear por tipo frásico, obtidas a partir de uma análise qualitativa dos dados obtidos.

Tabela 1: Tendência de padrões entoacionais do contorno nuclear dos tipos frásicos de interrogativas globais

\begin{tabular}{|c|c|c|c|c|}
\hline $\begin{array}{l}\text { Tipos } \\
\text { Frásicos } \\
\end{array}$ & Tendência EF & Tendência FC & Tendência MR & Semicontrolada \\
\hline GNI & $\mathrm{L}+\mathrm{H}^{*}(\mathrm{H}) \mathrm{L} \%$ & $\mathrm{~L}+(!) \mathrm{H}^{*} \mathrm{~L} \%$ & $\mathrm{~L}+(!) \mathrm{H}^{*} \mathrm{~L} \%$ & $\mathrm{~L}^{*}+\mathrm{H} \mathrm{L} \%$ \\
\hline GND & $\begin{array}{l}\mathrm{L}+(\mathrm{i}) \mathrm{H}^{*}(\mathrm{H} \%) /(\mathrm{H}-) \\
(!) \mathrm{H}+\mathrm{L}^{*} \mathrm{~L} \%\end{array}$ & $\begin{array}{l}\mathrm{L}+\mathrm{H}^{*}(\mathrm{HL}-)(i) \mathrm{H}+\mathrm{L}^{*} \\
\mathrm{~L} \%\end{array}$ & $\begin{array}{l}\mathrm{L}+(i) \mathrm{H}^{*}(\mathrm{HL}-) \mathrm{H}+\mathrm{L}^{*} \\
\mathrm{~L} \%\end{array}$ & $\mathrm{~L}+\mathrm{H}^{*} / \mathrm{H}+\mathrm{L} * / \mathrm{L} \%$ \\
\hline GNR & $\mathrm{L}^{*}+(\mathrm{i}) \mathrm{H} \mathrm{L} \%$ & $\mathrm{~L}+! \mathrm{H}^{*} \mathrm{~L} \% / ! \mathrm{H}+\mathrm{L} * \mathrm{~L} \%$ & $\begin{array}{l}\mathrm{L}+! \mathrm{H}^{*} \mathrm{~L} \% /(!) \mathrm{H}+\mathrm{L}^{*} \\
\mathrm{~L} \%\end{array}$ & - \\
\hline GAF & $\mathrm{L}+(\mathrm{i}) \mathrm{H}^{*}(\mathrm{H}) \mathrm{L} \%$ & $\begin{array}{l}\mathrm{L}+(!) \mathrm{H}^{*} \mathrm{~L} \% / \mathrm{H}^{*}+\mathrm{L} \\
\mathrm{L} \%\end{array}$ & $\mathrm{~L}+(!) \mathrm{H}^{*} \mathrm{~L} \%$ & - \\
\hline GAN & $\mathrm{L}+{ }_{i} \mathrm{H}^{*} \mathrm{~L} \%$ & $\mathrm{~L}+! \mathrm{H}^{*}(\mathrm{H}) \mathrm{L} \%$ & $\mathrm{~L}+! \mathrm{H}^{*} \mathrm{~L} \%$ & $\mathrm{~L}^{*}+\mathrm{H} \mathrm{L} \%$ \\
\hline GAC & $\mathrm{L}+{ }_{i} \mathrm{H}^{*}(\mathrm{~L}-) \mathrm{L} \%$ & $\mathrm{~L}+! \mathrm{H}^{*} \mathrm{~L} \% / ! \mathrm{H}+\mathrm{L}^{*} \mathrm{~L} \%$ & $\mathrm{~L}+\mathrm{H}^{*}(\mathrm{~L}-) \mathrm{L} \%$ & $\mathrm{~L}^{*}+\mathrm{H} \mathrm{L} \%$ \\
\hline GAT & $\mathrm{H}^{*}+\mathrm{L} \mathrm{L} \%$ & $\mathrm{H}^{*}+\mathrm{LL} \%$ & $\mathrm{H}^{*}+\mathrm{LL} \%$ & $\mathrm{~L}^{*}+\mathrm{H} \mathrm{L} \%$ \\
\hline GAE & $\mathrm{L}+\mathrm{H}^{*} \mathrm{H} \%$ & $\mathrm{~L}^{*}+\mathrm{H} \mathrm{H} \%$ & $\mathrm{~L}^{*}+\mathrm{H} \mathrm{H} \%$ & $\mathrm{~L}^{*}+\mathrm{H} / \mathrm{H} \%$ \\
\hline GAA & $\mathrm{L}+({ }) \mathrm{H}^{*}(\mathrm{H}) \mathrm{L} \%$ & $\mathrm{~L}+\left({ }_{\mathrm{i}}\right) \mathrm{H}^{*}(\mathrm{H}) \mathrm{L} \%$ & $\mathrm{~L}+\mathrm{H}^{*} \mathrm{~L} \%$ & $\mathrm{~L}^{*}+\mathrm{H} \mathrm{L} \%$ \\
\hline
\end{tabular}

$\mathrm{Na}$ Tabela 1, a coluna "Tipos Frásicos" apresenta as siglas referentes a cada tipo de interrogativa global investigada, conforme supracitado. As colunas "Tendência EF", "Tendência FC" e "Tendência MR" dizem respeito às produções de cada uma das três falantes, identificadas pelas siglas ER, FC e MR, do corpus de fala controlada. A coluna "Semicontrolada" diz respeito aos padrões entoacionais identificados para alguns dos tipos frásicos investigados a partir dos corpora do InAPoP.

Para GNI (interrogativas globais neutras de busca de informação), há semelhança na tendência encontrada entre as informantes de leitura controlada e as informantes de leitura semicontrolada. $\mathrm{O}$ padrão encontrado para o contorno nuclear foi $\mathrm{L}+\mathrm{H}^{*} \mathrm{~L} \%$, com a ressalva de que, para a segunda e a terceira falantes do corpus de leitura controlada, houve associação de downstep ao tom alto do contorno nuclear, na segunda e terceira repetições. Quanto ao contorno total das sentenças interrogativas globais de busca de informação, é possível representá-lo através da análise amostral da sentença "As alunas questionaram os jornalistas”, representada pela Figura 1. Nessa figura, é possível identificar quatro camadas, apresentadas pelo software Praat, as quais representam de cima para baixo: (a) o oscilograma em Hz, referente ao áudio da sentença realizada pela informante, (b) o espectrograma de som e a curva de $\mathrm{F}_{0}$, (c) a transcrição dos eventos tonais e (d) a representação ortográfica da sentença. 


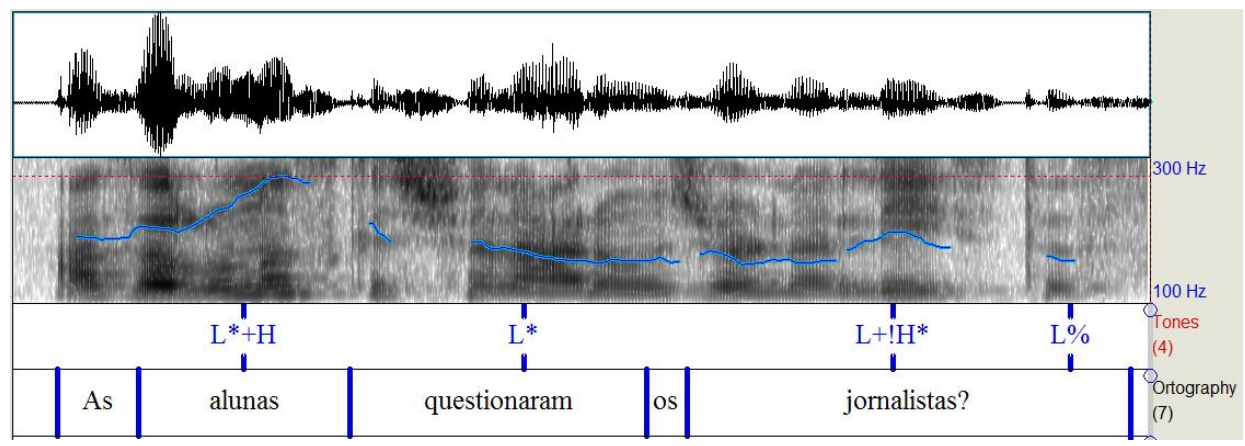

Figura 1. Análise entoacional da sentença "As alunas questionaram os jornalistas?", produzida por FC, no software Praat

Através da observação da Figura 1, é possível identificar um contorno nuclear ascendente $\left(\mathrm{L}+! \mathrm{H}^{*}\right.$ - associado à palavra mais à direita) e tom de fronteira baixo $(\mathrm{L} \%)$, o que confirma o padrão apontado por Frota et al. $(2011,2015)$ para interrogativas globais do PB. Esse contorno, obtido para fala controlada, assemelha-se ao contorno nuclear obtido em fala semicontrolada $\left(\mathrm{L}^{*}+\mathrm{H} \mathrm{L} \%\right)$. Na porção da sentença anterior ao contorno nuclear, observamos um tom ascendente inicial (acento tonal $\mathrm{L}^{*}+\mathrm{H}$ ), seguido por um tom baixo (acento tonal $\mathrm{L}^{*}$ ) que precede o tom nuclear.

Para GND (interrogativas globais neutras disjuntivas), há a necessidade de descrever dois contornos nucleares, cada um associado a um dos núcleos da disjunção. O primeiro contorno nuclear disjuntivo apresenta uma tendência de contorno entoacional ascendente que pode ser representada por $\mathrm{L}+(\mathrm{i}) \mathrm{H}^{*}$. Em algumas sentenças foi possível identificar acento frasal associado ao final do primeiro contorno nuclear, como é possível verificar na Figura 2 (HL-), que representa a sentença analisada "Você prefere uva ou banana?". Associada ao segundo contorno nuclear disjuntivo foi encontrada uma tendência de contorno entoacional descendente, representado por (!) $\mathrm{H}+\mathrm{L} * \mathrm{~L} \%$.

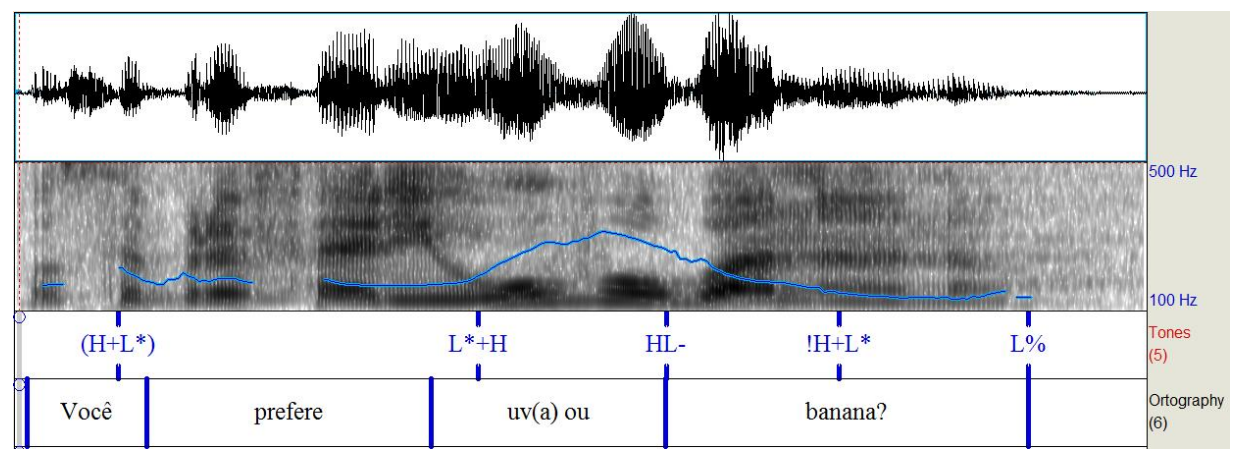

Figura 2. Análise entoacional da sentença "Você prefere uva ou banana?", produzida por MR, no software Praat

Quanto às sentenças do tipo GNR (interrogativas globais neutras retóricas), não há registro no corpus de leitura semicontrolada, portanto, só levamos em conta o observado nas produções controladas. Verificam-se duas possibilidades de contorno entoacional nuclear: ascendente e descendente. A característica que marca claramente esse tipo frásico é um rebaixamento de tessitura entoacional (representado pelo downstep (!) associado ao " $\mathrm{H}$ " do acento tonal nuclear) que precede o contorno nuclear, o qual se aproxima das sentenças neutras (FROTA; VIGÁRIO, 2000; TENANI, 2002) por apresentar, associado a ele, acento 
tonal descendente $\mathrm{H}+\mathrm{L}^{*}$. Dessa forma, o que caracteriza as interrogativas retóricas é esse rebaixamento, e não o contorno nuclear, como ocorre no caso das GNI, apontadas anteriormente. A Figura 3 apresenta o contorno total da sentença interrogativa global neutra retórica (GNR) "Será que fiz a melhor escolha?".

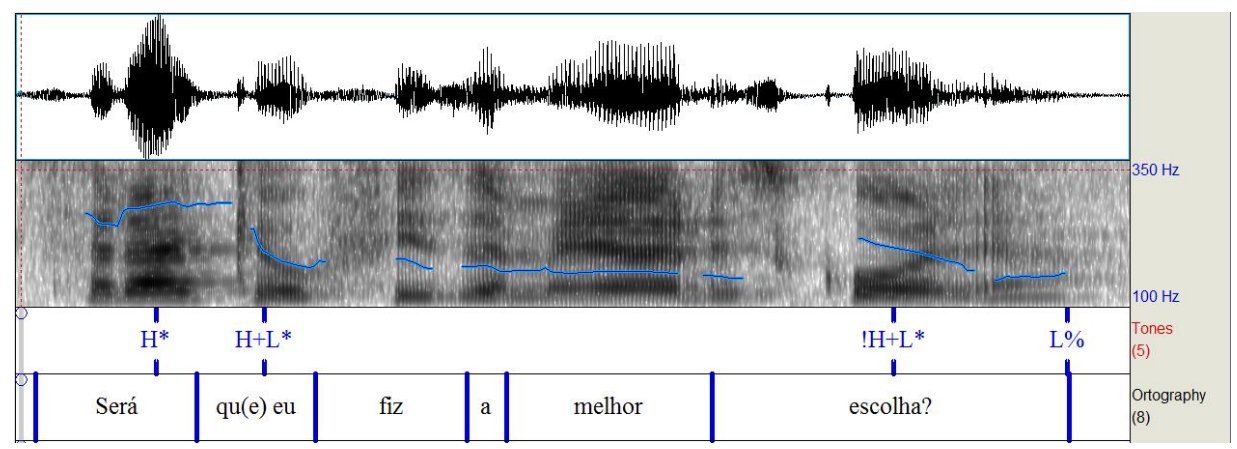

Figura 3. Análise entoacional da sentença "Será que eu fiz a melhor escolha?", produzida por MR, no software Praat

O contorno nuclear parece ter pouca relevância na caracterização do tipo frásico "interrogativas globais neutras retóricas". Por conta disso, não há uma preponderância clara de um contorno ascendente (44\% de ocorrências (soma das porcentagens apresentadas nas linhas 2, 5, 7, 8 e 11)) ou descendente (56\% de ocorrências (soma das porcentagens apresentadas nas linhas 3, 6, 9 e 10)) associado ao contorno nuclear desse tipo frásico, conforme é possível verificar na Tabela 2, abaixo.

Tabela 2: Frequência de ocorrência de contornos entoacionais nas sentenças interrogativas globais neutras retóricas.

\begin{tabular}{c|c|c}
\hline Contorno nuclear - GNR & Absoluto (Total: 36) & Relativo \\
\hline $\mathrm{L}+\mathrm{H}^{*} \mathrm{~L} \%$ & 8 & $22 \%$ \\
\hline$! \mathrm{H}+\mathrm{L} * \mathrm{~L} \%$ & 7 & $19 \%$ \\
\hline $\mathrm{L} * \mathrm{~L} \%$ & 6 & $17 \%$ \\
\hline $\mathrm{L}+\mathrm{H}^{*} \mathrm{~L} \%$ & 3 & $8 \%$ \\
\hline$! \mathrm{H}^{*}+\mathrm{L} \mathrm{L} \%$ & 3 & $8 \%$ \\
\hline $\mathrm{L}+\mathrm{H}^{*} \mathrm{~L} \%$ & 2 & $6 \%$ \\
\hline $\mathrm{L} *+j \mathrm{H} \mathrm{L} \%$ & 2 & $6 \%$ \\
\hline $\mathrm{H}^{*}+\mathrm{L} \mathrm{L} \%$ & 2 & $6 \%$ \\
\hline $\mathrm{H}+\mathrm{L}^{*} \mathrm{~L} \%$ & 2 & $6 \%$ \\
\hline $\mathrm{L}+\mathrm{H}^{*} \mathrm{~L} \%$ & 1 & $3 \%$ \\
\hline
\end{tabular}

Para GAF (interrogativas globais não neutras focalizadas), há uma tendência de contorno nuclear ascendente $\mathrm{L}+\mathrm{H}^{*} \mathrm{~L} \%$, exceto para a falante $\mathrm{FC}$ na terceira repetição. $\mathrm{O}$ que distingue o contorno entoacional total desse tipo frásico interrogativo é a presença de acento frasal associado à fronteira direita das palavras focalizadas, a depender do contexto de produção.

Os tipos frásicos GAN (interrogativas globais não neutras negativas) e GAC (interrogativas globais não neutras confirmatórias) apresentam um padrão entoacional nuclear bastante semelhante ao que foi apontado para GAF, que pode ser descrito por $\mathrm{L}^{*}+(!) \mathrm{H} \mathrm{L} \%$, 
com a exceção das tendências encontradas para a falante FC que, novamente, destoou do observado em relação às demais falantes, por apresentar um contorno nuclear descendente para esse tipo frásico. Assim como no caso das interrogativas focalizadas, o que caracteriza tanto as interrogativas negativas quanto as confirmatórias é a presença de tom relacionado à fronteira (acento frasal ou tom de fronteira) associado à fronteira direita da palavra focalizada (palavra foco da negação, no caso das interrogativas negativas, ou palavra a ser confirmada, no caso das interrogativas confirmatórias) ou a presença do acento tonal $\mathrm{H}^{*}+\mathrm{L}$ associado a essa palavra. Nossos resultados de marcação de foco em sentenças interrogativas globais vão ao encontro do que afirma Fernandes (2007a, 2007b) sobre a marcação de foco em sentenças declarativas do PB. Segundo essa autora, elementos focalizados em PB são marcados entoacionalmente por presença de acento frasal associado a sua fronteira direita ou por acento tonal $\mathrm{H}^{*}+\mathrm{L}$ associado à sílaba tônica desses elementos.

A Figura 4 representa a análise da sentença interrogativa global não-neutra focalizada (GAF) "Foi o Pedro que chegou por último?", em que a focalização ocorre nas palavras "foi”, "Pedro" e "último", e exemplifica o que acabamos de expor.

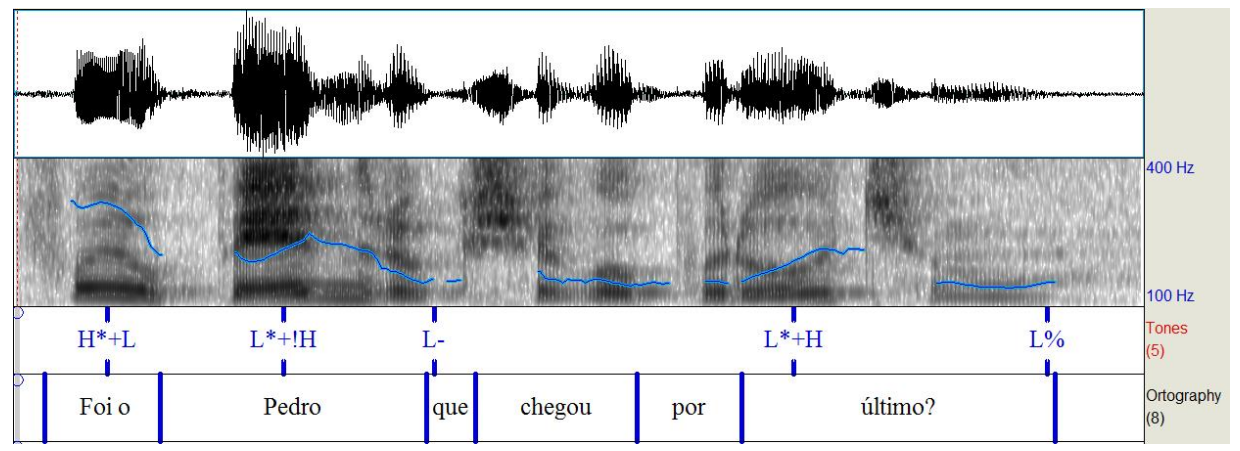

Figura 4. Análise entoacional da sentença "Foi o Pedro que chegou por último?”, produzida por MR, no software Praat

Para GAT (interrogativas globais não neutras eco manipulatórias), encontramos, em fala controlada, um padrão claramente descendente do contorno nuclear, marcado como $\mathrm{H}^{*}+\mathrm{L} \mathrm{L} \%$, enquanto que, para a fala semicontrolada, a configuração tonal observada para o contorno nuclear foi $\mathrm{L}^{*}+\mathrm{H} \mathrm{L} \%$. A Figura 5 ilustra o contorno total encontrado para esse tipo frásico.

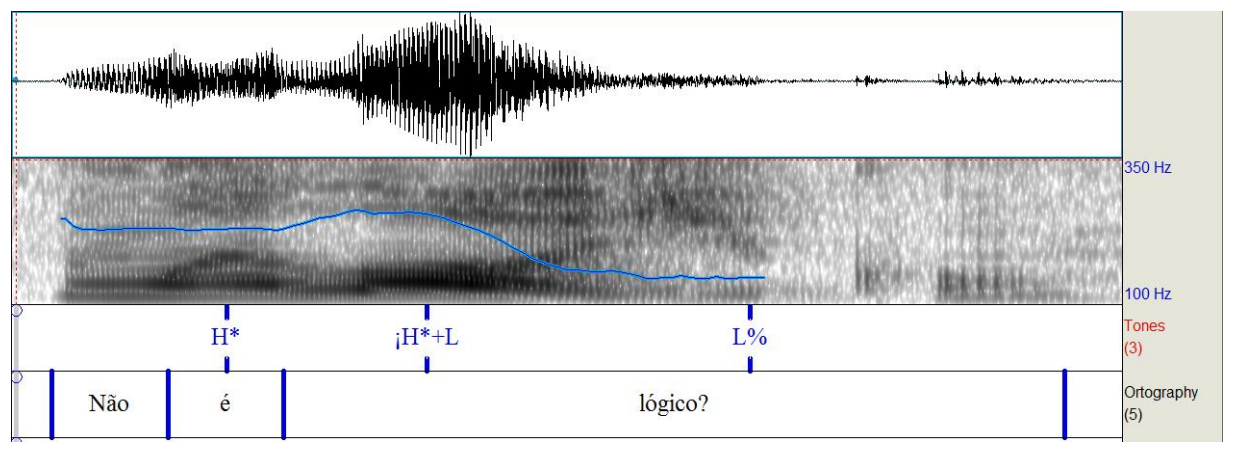

Figura 5. Análise entoacional da sentença "Não é lógico?", produzida por MR, no software Praat 
Por sua vez, em sentenças classificadas como GAE (interrogativas globais não neutras eco geral), há uma tendência melódica clara ascendente do contorno nuclear, descrita como $\mathrm{L}^{*}+\mathrm{H} \mathrm{H} \%$, tanto para a fala semicontrolada quanto para a controlada. Tal descrição é relevante, pois, categoricamente, distingue esse tipo frásico das interrogativas eco manipulatórias, que, apesar de também serem classificadas como eco por, pragmaticamente, representarem repetição de uma informação no contexto, estão associadas a um contorno entoacional radicalmente diferente $\left(\mathrm{H}^{*}+\mathrm{L} \mathrm{L} \%\right.$, observado em fala controlada) do observado para as interrogativas eco geral $\left(\mathrm{L}^{*}+\mathrm{H} \mathrm{H} \%\right)$. O contorno típico associado a sentenças do tipo frásico GAE pode ser observado no exemplo representado pela Figura 6.

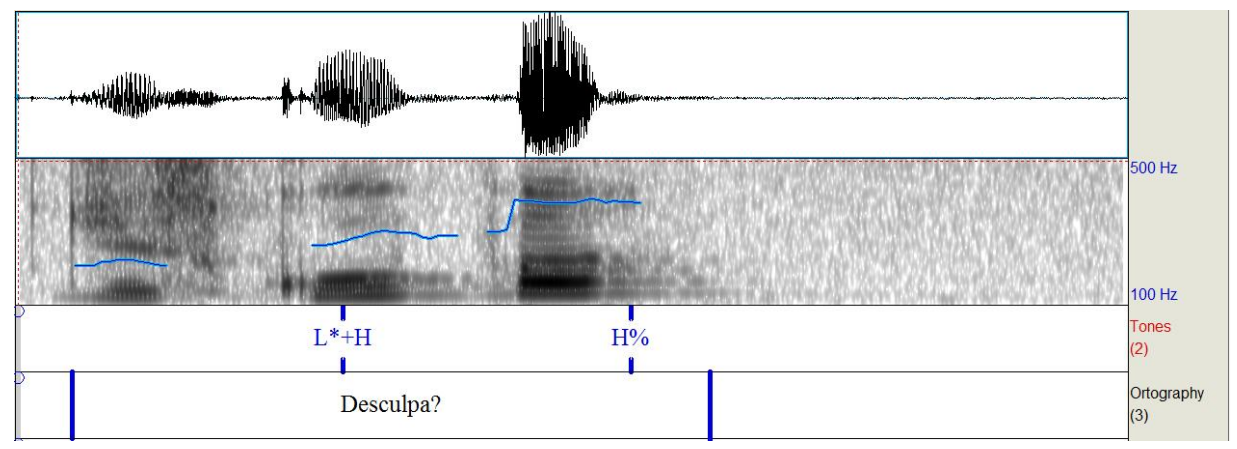

Figura 6. Análise entoacional da sentença "Desculpa?”, produzida por EF, no software Praat

Finalmente, para o tipo frásico GAA (interrogativas globais não neutras eco absoluta antiexpectativa), é possível identificar, para o contorno nuclear, o padrão melódico $\mathrm{L}^{*}+(i) \mathrm{H}$ $\mathrm{L} \%$ para fala controlada e $\mathrm{L}^{*}+\mathrm{H} \mathrm{L} \%$ para fala semicontrolada. Ao observarmos o contorno total desse tipo frásico, verificamos, de maneira geral, um upstep no contorno nuclear como elemento caracterizador desse tipo frásico, conforme pode ser observado na Figura 7, que representa a análise da sentença GAA “Vou ter que esperar o próximo?”.

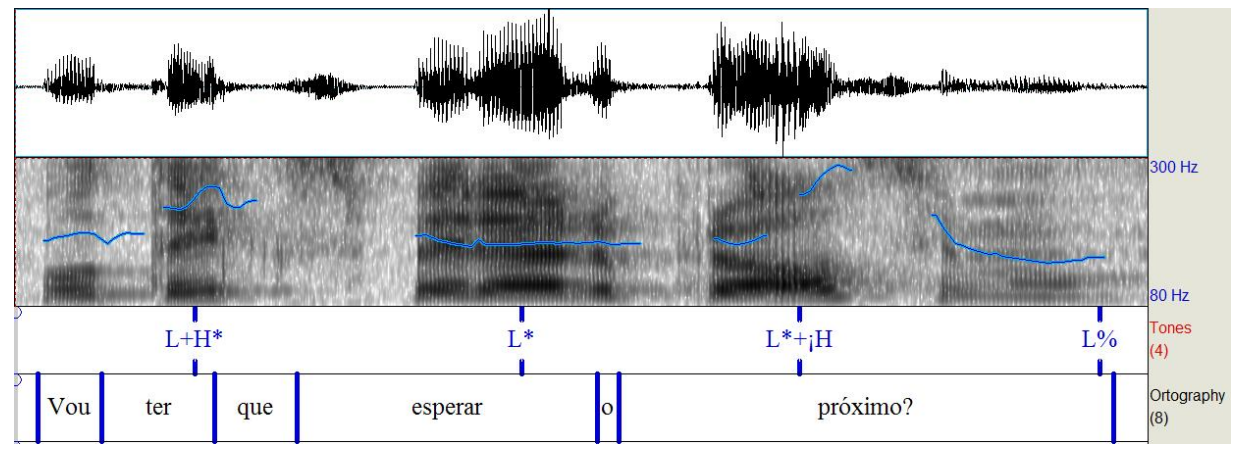

Figura 7. Análise entoacional da sentença "Vou ter que esperar o próximo?", produzida por MR, no software Praat 


\section{Considerações finais}

É possível observar tendências no padrão entoacional das interrogativas e variações em F0 a depender do tipo de interrogativa.

Tanto o acento tonal nuclear quanto o tom de fronteira são imprescindíveis para a caracterização de cada tipo frásico, conforme foi evidenciado pela descrição dos contornos nucleares dos tipos frásicos investigados. Tomando a interrogativa global não-neutra eco geral (GAE) como um exemplo, sua distinção se dá justamente no tom de fronteira alto, em todas as repetições, tanto na fala controlada quanto na semicontrolada.

A importância do contorno total na caracterização dos diferentes tipos frásicos pode ser comprovada principalmente pelas análises das interrogativas globais neutras retóricas (GNR) e dos tipos frásicos em que há focalização (interrogativas não neutras focalizadas GAF -, negativas - GAN - e confirmatórias - GAC), nos quais os eventos tonais associados ao contorno total são caracterizadores dos tipos frásicos. No caso das interrogativas neutras retóricas (GNR), o rebaixamento da tessitura associado ao contorno total é o que caracteriza esse tipo frásico, e não apenas seu contorno nuclear, o qual pode ser ascendente ou descendente. Já para os tipos em que há focalização, por exemplo, GAF, GAN e GAC, o elemento caracterizador é a presença de acento frasal associado à fronteira direita do elemento focalizado. Verifica-se, portanto, que há diferenças na atribuição de eventos tonais (acento tonal nuclear, tom de fronteira e acento frasal), a depender do tipo de interrogativa, em relação ao contorno total, o que comprova a hipótese apresentada neste trabalho.

Cabe acrescentar que, em etapas posteriores de nossa pesquisa, buscaremos: (a) aprofundar a descrição das interrogativas, ampliando o corpus, de modo a incluir as interrogativas parciais; (b) relacionar a atribuição dos eventos tonais à formação de domínios prosódicos, conforme a teoria da Fonologia Prosódica (SELKIRK, 1984, 1986, 2000; NESPOR; VOGEL, 1986, 2007); e (c) investigar se as diferenças observadas em relação aos eventos tonais associados aos diferentes tipos de interrogativa podem ser percebidas por falantes nativos do dialeto paulista do PB, através de testes de percepção.

\section{REFERÊNCIAS}

BOERSMA, P.; WEENINK, D. Praat: Doing Phonetics by Computer [Computer program]. Version 5.3. 82. 2014. Disponível em: <http://www.fon.hum.uva.n1/praat/>. Acesso em: 27 jun. 2015.

CRUZ, M.; FROTA, S. Prosódia dos tipos frásicos em variedades do Português Europeu: produção e percepção. Textos Seleccionados, XXVI Encontro da Associação Portuguesa de Linguística, Lisboa, APL, 2011, p. 208-225.

FERNANDES, F. R. Tonal association in neutral and subject-narrow-focus sentences of Brazilian Portuguese: a comparison with European Portuguese. Journal of Portuguese Linguistics, v.6, n.1, p. 91-115, 2007a. 
. Ordem, focalização e preenchimento em português: sintaxe e prosódia. 2007b. 415 p. Tese (Doutorado em Linguística) - Instituto de Estudos da Linguagem, Universidade Estadual de Campinas, Campinas.

FROTA, S. (Coord.). InAPoP - Interactive Atlas of the Prosody of Portuguese. Projeto de investigação científica (processo FCT PTDC/CLE-LIN/119787/2010). Universidade de Lisboa, 2012-2014.

FROTA, S.; CRUZ, M.; SVARTMAN, F.; VIGÁRIO, M.; COLLISCHONN, G.; FONSECA, A.; SERRA, C.; SCHWINDT, L. C. Portuguese: Labelling intonational variation across varieties of European and Brazilian Portuguese. Workshop on Romance ToBI, Universitat Rovira i Virgili, 2011.

FROTA, S.; CRUZ, M.; FERNANDES-SVARTMAN, F.; COLLISCHONN, G.; FONSECA, A.; SERRA, C.; OLIVEIRA, P.; VIGÁRIO, M. Intonational variation in Portuguese: European and Brazilian varieties. In: FROTA, S.; PRIETO, P. (Eds.). Intonational variation in Romance. Oxford: Oxford University Press, 2015. p. 235-283. Disponível em: <http://labfon.letras.ulisboa.pt/InAPoP/resources.html>. Acesso em: 15 jun. 2015.

FROTA, S.; VIGÁRIO, M. Aspectos de prosódia comparada: ritmo e entoação no PE e no PB. In: CASTRO, R. V.; BARBOSA, P. (Orgs.). Actas do XV Encontro Nacional da Associação Portuguesa de Linguística. v.1. Coimbra: Associação Portuguesa de Linguística, 2000, p. 533-555. Disponível em: <http//labfon.letras.ulisboa.pt/InAPoP/resources.html>. Acesso em: 15 jun. 2015.

JUN, S-A. Prosodic Typology - The Phonology of Intonation and Phrasing. New York: Oxford University Press, 2005. 474 p.

LADD, D. R. Intonational Phonology. Cambridge: Cambridge University Press, 1996. 334 p. 2008. 372 p.

Intonational Phonology, $2^{\text {nd }}$ edition. Cambridge: Cambridge University Press,

MATEUS, M. H. M.; BRITO, A. M.; DUARTE, I.; FARIA, I. H.; FROTA, S.; MATOS, G.; OLIVEIRA, F.; VIGÁRIO, M.; VILLALVA, A. Gramática da Língua Portuguesa. 5. ed. Lisboa: Ed. Caminho, 2003. p. 460-479.

MORAES, J. The Pitch Accents in Brazilian Portuguese: analysis by synthesis. Laboratório de Fonética Acústica, Faculdade de Letras UFRJ/Faculdade de Letras UFRJ/CNPq, 2008. 9 p.

NESPOR, M.; VOGEL, I. Prosodic Phonology. Dordrecht: Foris Publications, 1986. 327p. Prosodic Phonology: with a new foreword. Berlin: Mouton de Gruyter, 2007. $327 \mathrm{p}$.

PIERREHUMBERT, J. The phonology and phonetics of English intonation. 1980. 201 p. Tese (Doutorado em Filosofia) - Massachusetts Institute of Technology, Cambridge, Mass. 
PIERREHUMBERT, J.; BECKMAN, M. Japanese Tone Structure. Cambridge, Mass: The Massachusetts Institute of Technology Press, 1988. 280 p.

SELKIRK, E. O. Phonology and syntax: The relation between sound and structure. Cambridge: The Massachusetts Institute of Technology Press, 1984. 424 p.

On derived domains in sentence phonology. Phonology Yearbook, n. 3, p. 371405, 1986.

The interaction of constraints on prosodic phrasing. In: HORNE, M. (Ed.). Prosody:

Theory and Experiment. Netherlands: Kluwer Academic Publishers, 2000. p. 231-261.

SERRA, C. R. Realização e percepção de fronteiras prosódicas no português do Brasil: fala espontânea e leitura. 2009. 241 p. Tese (Doutorado em Letras Vernáculas) Universidade Federal do Rio de Janeiro, Rio de Janeiro.

TENANI, L. E. Domínios prosódicos no português do Brasil: implicações para a prosódia e para a aplicação de processos fonológicos. 2002. 317 p. Tese (Doutorado em Linguística) - Universidade Estadual de Campinas, Campinas.

TRUCKENBRODT, H.; SANDALO, M. F. S.; ABAURRE M. B. M. Elements of Brazilian Portuguese intonation. Journal of Portuguese Linguistics, v. 8, p. 75-114, 2009.

VIGÁRIO, M.; FERNANDES-SVARTMAN, F. R. A atribuição de acentos tonais em compostos no português do Brasil. In: BRITO, A. M.; SILVA, F.; VELOSO, J.; FIÉIS, A. (Orgs.). XXV Encontro da Associação Portuguesa de Lingüística - Textos Seleccionados. Porto: Tip. Nunes, Ltda - Maia, 2010, v. 1, p. 769-786.

Recebido em: 03/05/2016

Aprovado em: 24/05/2016 